\title{
Non-stationary Signal Analysis using TVAR Model
}

\author{
G. Ravi Shankar Reddy ${ }^{1}$ and Dr. Rameshwar Rao ${ }^{2}$ \\ ${ }^{1}$ Dept.of ECE, CVR College of Engineering, Hyderabad, India \\ ${ }^{2}$ Vice - Chancellor, JNT University, Hyderabad, India \\ ${ }^{1}$ ravigosula_ece39@yahoo.co.in, ${ }^{2}$ rameshwar_rao@hotmail.com
}

\begin{abstract}
In this paper Time-varying Auto regressive model (TVAR) based approach for instantaneous frequency(IF) estimation of the frequency modulated signal in noisy environment is presented. Covariance method is applied for least square estimation of timevarying autoregressive parameters. Time-varying parameters are expressed as a linear combination of constants multiplied by basis functions. Then, the time-varying frequencies are extracted from the time-varying parameters by calculating the angles of the estimation error filter polynomial roots. The experimental results are presented for IF estimation, prediction and power spectrum estimation of non stationary signals. we have also discussed the spectral resolution ability of TVAR Model. Simulation and experimental results demonstrate that TVAR is an effective solution to non-stationary signal analysis and has strong capability in signal time-frequency feature extraction.
\end{abstract}

Keywords: Instantaneous frequency estimation, basis functions, noisy environment, prediction, powerspectrum, spectralresolution

\section{Introduction}

Frequency modulated (FM) signal estimation in a noisy environment is important for many commercial and military applications. These signals are analyzed in engineering applications such as telecommunications, biomedical engineering, radar, sonar and signal processing.

A time-varying autoregressive (TVAR) approach is used for modeling nonstationary signals, and frequency information is then extracted from the TVAR parameters. Two methods are used for estimating the TVAR parameters [1]: the adaptive algorithm approach and the basis function approach. Adaptive algorithms, such as the least mean square (LMS) and the recursive least square (RLS), use a dynamic model for adapting the TVAR parameters and are capable of tracking time-varying frequency, provided that the variation is slow [4].

The basis function method employs an explicit model for the TVAR parameter variation, and model parameters are estimated via a block calculation where parameters are expanded as a summation of the weighted time-functions [2], are capable of tracking both the fast or the slow time-varying frequencies .However, the selection of the expansion dimension and the basis function is questionable since there is no fundamental theorem on how to choose them [2]. We propose here to apply traditional polynomial functions (namely time polynomial, Legendre polynomials, and Chebyshev polynomials) because their realization is easy, and they can fairly accurate a broad range of variations. In this article, we employ basis functions that are powers of the time variable $\mathrm{n}$ (time basis function).

The paper is presented as follows. It explains the Time-varying Autoregressive modeling in Section 2. In Section 3 it explains the selection of basis function and TVAR model order determination by means of Maximum likelihood estimator. In Section 4 it gives Time varying 
power spectrum estimation using TVAR Model. Experimental procedure is given in Section 5. Simulation results are presented in Section 6. Concluding remarks are given in Section 7.

\section{TVAR Modeling}

The non stationary discrete-time stochastic process $x_{n}$ is represented by $\mathrm{p}^{\text {th }}$ order TVAR model as

$x_{n}=-\sum_{k=1}^{p} a_{k, n} x_{n-k}+v_{n}$

Here $a_{k, n}$ are time-varying coefficients and $v_{n}$ is a stationary white noise process and whose mean is zero and variance is $\sigma_{v}{ }^{2}$. According to the time -varying coefficients evolution, TVAR is likely to be categorized in to two group's i.e. adaptive method and basis function approach.

TVAR model based on the basis function technique is able to trace a strong non-stationary signal, that's why this model is focused in the present study. In this technique, each of its time-varying coefficients are modeled as linear combination of a set of basis functions [9].

The purpose of the basis is to permit fast and smooth time variation of the coefficients. If we denote $u_{m, n}$ as the basis function and consider a set of $(q+1)$ function for a given model, we can state the TVAR coefficients in general as

$a_{k, n}=\sum_{m=0}^{q} a_{k m} u_{m, n}$

From (2) we examine that, we have to calculate the set of parameters $a_{k m}$ for $\left\{\mathrm{k}=1,2, \ldots \ldots \ldots . \mathrm{p} ; \mathrm{m}=0,1,2, \ldots \ldots \ldots \ldots, \mathrm{q} ; a_{0 m}=1\right\}$ in order to compute the TVAR coefficients $a_{k, n}$,and the TVAR model is absolutely specified by this set.

The TVAR coefficients are designed as follows, we consider single realization of the process $x_{n}$.For a given realization of $x_{n}$ we can analyze (1) as a time-varying linear prediction error filter and consider $v_{n}$ to be the prediction error

$v_{n}=x_{n}-\hat{x}_{n}$

Where

$\hat{x}_{n} \triangleq-\sum_{k=1}^{p} a_{k, n} x_{n-k}$

The total squared prediction error, which is as well as the error in modeling $x_{n}$, is now specified by

$\epsilon_{p}=\sum_{\tau}\left|v_{n}\right|^{2}$

Substitute (2) in (4) and the prediction error $v_{n}$ can be written as 
$v_{n}=x_{n}+\sum_{k=1}^{p} \sum_{m=0}^{q} a_{k m} u_{m, n} x_{n-k}$

The total squared prediction error can be formulated as

$\epsilon_{p}=\sum_{\tau}\left|x_{n}+\sum_{k=1}^{p} \sum_{m=0}^{q} a_{k m} u_{m, n} x_{n-k}\right|^{2}$

For modeling the non stationary stochastic process $x_{n}$, using covariance technique, we make no assumptions on the data outside [0, N-1]. In equation (6) $\tau$ is the interval over which the summation is performed and set $\tau=[p, N-1]$. By minimizing the mean squared prediction error in (6) we can estimate the time-varying parameters $a_{k m}$ [10]. We can minimize the mean squared prediction error in (6) by means of setting the gradient of $\epsilon_{p}$ with respect to $a_{l g}^{*}$ zero

$\frac{\partial \epsilon_{p}}{\partial a_{l g}^{*}}=\sum_{\tau} \frac{\partial v_{n} v_{n}^{*}}{\partial a_{l g}^{*}}=\sum_{\tau} v_{n} \frac{\partial v_{n}^{*}}{\partial a_{l g}^{*}}=0$

$\{l=1,2, \cdots, p ; g=0,1, \cdots, q\}$

Where

$v_{n}^{*}=x_{n}^{*}+\sum_{l=1}^{p} \sum_{g=0}^{q} a_{l g}^{*} u_{g, n}^{*} x_{n-l}^{*}$

And the derivative of $v_{n}^{*}$ with respect to $a_{l g}^{*}$

$\frac{\partial v_{n}^{*}}{\partial a_{l g}^{*}}=u_{g, n}^{*} x_{n-l}^{*}$

Consequently (7) becomes,

$\sum_{\tau} v_{n} u_{g, n}^{*} x_{n-l}^{*}=0$

The above mentioned condition is similar to the orthogonality principle encountered in stationary signal modeling. Substitute (5) in (8) we have

$\sum_{\tau}\left(x_{n}+\sum_{k=1}^{p} \sum_{m=0}^{q} a_{k m} u_{m, n} x_{n-k}\right) u_{g, n}^{*} x_{n-l}^{*}=0$

Now we define a function $c_{m g}(l, k)$ as shown below,

$c_{m g}(l, k) \triangleq \sum_{\tau} u_{m, n} x_{n-k} u_{g, n}^{*} x_{n-l}^{*}$

Using the above definition in (9) we have, 
$\sum_{k=1}^{p} \sum_{m=0}^{q} a_{k m} c_{m g}(l, k)=-c_{0 g}(l, 0)$

The above equation represents a system of $p(q+1)$ linear equations. The above system of linear equations can be efficiently represented in matrix form as follows.

Define a column vector $a_{m}$ as follows

$a_{m}=\left[\begin{array}{llll}a_{1 m} & a_{2 m} & \cdots & a_{p m}\end{array}\right]^{T}$,

where $m=0,1, \cdots, q$

We can use the function (10) to find the following matrix for $0 \leq(m, g) \leq q$

$C_{m g}=\left[\begin{array}{cccc}c_{m g}(1,1) & c_{m g}(1,2) & \cdots & c_{m g}(1, p) \\ c_{m g}(2,1) & c_{m g}(2,2) & \cdots & c_{m g}(2, p) \\ \vdots & & \ddots & \vdots \\ c_{m g}(p, 1) & c_{m g}(p, 2) & \cdots & c_{m g}(p, p)\end{array}\right]$

The above matrix is of size pxp and all the different values for $\mathrm{m}$ and $\mathrm{g}$ resulting in $(\mathrm{q}+1) \mathrm{x}(\mathrm{q}+1)$ such matrices, by means of these matrices, we can now describe a block matrix as shown below,

$C=\left[\begin{array}{cccc}C_{00} & C_{01} & \cdots & C_{0 q} \\ C_{10} & C_{11} & \ddots & C_{1 q} \\ : & : & : & : \\ \cdot & \cdot & \cdot & \cdot \\ C_{q 0} & C_{q 1} & \cdots & C_{q q}\end{array}\right]$

The above Block matrix $\mathrm{C}$ has $(\mathrm{q}+1) \mathrm{x}(\mathrm{q}+1)$ elements and each element is a matrix of size pxp, which implies the Block matrix $C$ of size $p(q+1) x p(q+1)$.

Now we describe a column vector $d_{m}$ as shown below

$d_{m}=\left[\begin{array}{cccc}c_{0 m}(1,0) & c_{0 m}(2,0) & \cdots & c_{0 m}(p, 0)\end{array}\right]^{T}$

where $m=0,1, \cdots, q$

By using the definitions from (12)-(15) we can represent the system of linear equations in (11) in a compact matrix form as follows

$$
\underbrace{\left[\begin{array}{ccc}
C_{00} & \cdots & C_{0 q} \\
\vdots & \ddots & \vdots \\
C_{q 0} & \cdots & C_{q q}
\end{array}\right]}_{C} \underbrace{\left[\begin{array}{c}
a_{0} \\
\vdots \\
a_{q}
\end{array}\right]}_{a}=-\underbrace{\left[\begin{array}{c}
d_{0} \\
\vdots \\
d_{q}
\end{array}\right]}_{d}
$$

$C a=-d$ 
The above equations reduce to the Yule-walker equations (YWE) for a stationary AR model, as soon as $\mathrm{q}=0$. By solving the above matrix equation, we can obtain the set of TVAR parameters $a_{k m}$ (elements of $a$ ), the predictor coefficients $a_{k, n}$ can now be calculated using (2)

\section{Parameter Selection}

The TVAR parameter selection is basically depends on three degrees of freedom, such as the TVAR order $\mathrm{p}$, the basis function order $\mathrm{q}$, and the set of basis functions $u_{m, n}$.

\subsection{Choice of the Basis Functions}

The basis functions $u_{m, n}$ must be independent and non-zero for $\mathrm{n}=0,1, \ldots . \mathrm{N}-1$, and $u_{m, n}=1$ ,if $n=0$.If a priori information about the signal variation is known, the basis functions should be chosen such that the trends in parameter change is retained. In case, when a priori information is unavailable selection of basis is trial and error.

According to equation 2, no particular constraint is imposed on the basis $u_{m, n}$, consequently one will be able to track only variations which are approximable by this set of functions. Numerous solutions have been projected in the literature such as time basis functions, Legendre polynomial, Chebyshev polynomial, Discrete prolate spheroidal (DPSS) sequence, Fourier basis, Discrete cosine basis, Walsh basis, Multi wavelet basis, none of these solutions seems to be perfect, since the selection of $u_{m, n}$ desires some priori information upon the time variations present in $x_{n}$. Then again, basis such as prolate spheroidal functions are extremely tough to generate. We propose here to apply traditional polynomial functions (namely time polynomial, Legendre polynomials, and Chebyshev polynomials) since their realization is easy, and they can fairly accurate a broad range of variations. In this article, we employ basis functions that are powers of the time variable $n$ (time basis function) as given below,

$u_{m, n}=\left(\frac{n-p}{N}\right)^{m}$

$m=0,1, \ldots \ldots \ldots \ldots q$;

$n=p, p+1, \ldots \ldots . . . N-1$.

$\mathrm{N}$ denotes the length of the data record that is being modeled.

\subsection{Order Selection}

In the presence of noise The TVAR model can distinguish several time-varying spectral peaks well. However it is sensitive to model order change. False spectral peaks may be produced by the TVAR modeling approach, when an erroneous model order is chosen. Thus, the determination of right model order in TVAR modeling is a significant issue. There are few techniques in choice of TVAR model order. For instance, Bayesian technique [7] and Akaike information criterion (AIC) [7] are used for the determination of model orders in TVAR models. In this article, we consider the choice of the model order as a Maximumlikelihood (ML) estimation [8] technique. In this technique, by maximizing the likelihood function we can determine the model order. 


\section{Maximum Likelihood Estimation (MLE)}

The TVAR Model for the non stationary discrete-time stochastic process $x_{n}$ is

$x_{n}=-\sum_{k=1}^{p} \sum_{m=0}^{q} a_{k m} u_{m, n} x_{n-k}+v_{n}$

The above can be represented in compact form as

$$
x_{n}=-Z^{T}[n] a+v_{n}
$$

Where $Z[n]$ is

$Z[n]$

$=\Phi[n] \otimes u[n]$

Here, $\otimes$ denote Kronecker multiplication.

$$
\begin{gathered}
\Phi[n]=\left[x_{n-1}, x_{n-2} \ldots \ldots x_{n-p}\right]^{T} \\
u[n]=\left[u_{0 n}, u_{1 n} \ldots \ldots u_{q n}\right]^{T}
\end{gathered}
$$

Moreover

$a=\left[a_{1}^{T}, a_{2}^{T} \ldots \ldots a_{p}^{T}\right]$

Here

$a_{k}^{T}=\left[a_{k 0}, a_{k 1} \ldots \ldots a_{k q}\right]$

Step-1: compute

$Z[n]=\Phi[n] \otimes u[n]$

Step-2: calculate

$C=-\left(\sum_{n=p}^{N} Z[n] Z^{T}[n]\right)^{-1}\left(\sum_{n=p}^{N} Z[n] x[n]\right)$

Step-3: Estimate

$\hat{\beta}=\frac{1}{N} \sum_{n=0}^{N}\left[x_{n}-C^{T} Z[n]\right]^{2}$

Step-4: Obtain the cost function

$J(p, q)=\frac{p(q+1)+2-N}{2} \log (2 \pi \hat{\beta})-\frac{1}{2} \log \left|\sum_{n=0}^{N} Z[n] Z^{T}[n]\right|$ 
Step-5: Maximize the above cost function to select the expansion dimension $\mathrm{q}=q_{\text {opt }}$ and the model order $\mathrm{p}=p_{\text {opt }}$, where $p_{\text {opt }} \in\left\{1,2,3,4 \cdots \cdots p_{\max }\right\}$ and $q_{\text {opt }} \in\left\{0,1,2,3,4 \cdots \cdots q_{\max }\right\}$

\section{Time-Varying Frequency/Time-varying Power Spectra}

The representation of a nonstationary signal in the time-frequency domain by estimating time-varying spectrum show characteristics which are not normally observed in the timedomain. There are many known approaches in estimating time-varying power spectral density and obtain a non-negative time-frequency distribution. Good review on the time-frequency distribution can be found[15].For example, spectrogram obtained as a squared magnitude of the short-time Fourier transform(STFT)[15] have been used in representing non-stationary signals such as speech. Wigner-Ville [17] distribution is computed as a Fourier transform of the instantaneous correlation function defined as $x(\mathrm{t}+\tau / 2) x^{*}(\mathrm{t}-\tau / 2)$ and have been studied by many researchers ,but it also generates spurious spectra caused by cross-terms when more than one spectral peak exist. Cohen [15] studied a family of time-frequency distribution and investigated desired properties of time-frequency distribution. A Cohen's general class of time-frequency distribution with exponential Kernel is suggested by Choi and Williams [16] for the estimation of time-varying power spectrum with reduced cross terms.

Although autoregressive modeling approaches have been used for resolving multiple spectral peaks closely separated(super-resolution spectral estimation)in stationary processes, there has been very little study in estimating time-varying spectra using a TVAR modeling approach. It is mainly because the TVAR modeling approach is sensitive to incorrect selection of model order, and can result spurious spectral peaks if the model order is not correctly selected. The MLE approach selected correct model order in the experiment with synthesized data, and TVAR model approach is comparable (or)better in estimating timevarying spectra.

The time-varying frequency can be extracted from the TVAR parameters $a_{k, n}$ since the non stationary signal is modeled as the output of the TVAR process, with zero-mean white noise input $v_{n}$.The time varying power spectral density can be estimated from time varying parameter $a_{k, n}$ as follows [8]

$$
\mathrm{P}(\mathrm{f} ; \mathrm{n})=\frac{\sigma^{2}}{\left|1+\sum_{k=1}^{p} a_{k, n} e^{-j 2 \pi f k}\right|^{2}}
$$

Where $a_{k, n}$ are TVAR parameters, and $\sigma^{2}$ is

$$
\sigma^{2}=\frac{1}{N-p} \sum_{n=p+1}^{N-1}\left|x_{n}+\sum_{k=1}^{p} a_{k, n} x_{n-k}\right|^{2}
$$

Time-Varying frequencies can be extracted from the time-varying power spectrum by locating the peak of the P (f; $n$ ). If there are $p$ frequency components in the signal, one can chose the frequency estimate as the locations of the $\mathrm{p}$ largest spectral peaks. Note that a threshold could be also set so that the peak below this threshold would be considered as that of an additive noise. Another way to extract the time-varying frequency from the TVAR is by calculating the roots $\mathrm{Z}_{\mathrm{k}}$ of the estimation error filter polynomial

$z^{p}+a_{1, n} z^{p-1}+a_{2, n} z^{p-2}+a_{3, n} z^{p-3}+\ldots \ldots . .+a_{p, n}=0$ 
and choosing the frequency estimate as the angles of the roots $\mathrm{z}_{\mathrm{k}}$. For real signal, the roots may be complex conjugate to each other. so only the roots in either upper(or)lower half of complex plane are selected. For example, if the number of the frequency components is $\mathrm{p}$, the closest $\mathrm{p}$ poles to the unit circle in the upper half of the z-plane are chosen. For complex case consider the poles that are close to unit circle. The time-varying frequencies $f_{i, n}$ are then

calculated by $f_{i, n}=\frac{\arg \left[P_{i, n}\right]}{2 \pi}$, where $P_{i, n}$ are the time-varying poles of the above polynomial. According to kay[18]estimating the frequency in this way is slightly more accurate than locating the frequency as the largest spectral peak location.

\section{Experimental Procedure}

Step1: Compute TVAR model order $\mathrm{p}$ and $\mathrm{q}$ using MLE algorithm, choose the basis function $u_{m, n}, \mathrm{~m}=1,2 \ldots \ldots$. $\mathrm{n}=1,2 \ldots \mathrm{N}$.

Step2:For covariance technique of signal modeling set $\tau=[p, N-1]$ and compute $c_{m g}(l, k)$ by means of equation (10) to find the matrix $C_{m g}$ in (13), subsequently, set up the matrix $\mathrm{C}$ in (14), as well, use $c_{m g}(l, k)$ to calculate $d_{m}$ in (15) .

Step3: Calculate the TVAR parameters $a_{k m}$ by solving $\mathrm{C} a=-\mathrm{d}$ in (16) and form the coefficients $a_{k, n}$ using (2).

Step4: Solve the roots of the time-varying auto regressive polynomial formed by TVAR linear prediction filter $\mathrm{A}(\mathrm{z} ; \mathrm{n})=1+\sum_{k=1}^{p} a_{k, n} z^{-k}$ at each instant $\mathrm{n}$ to find the time-varying poles: $P_{i, n}, \mathrm{i}=1,2 \ldots . . \mathrm{p}$.

Step5: The instantaneous frequency of the non stationary signal for each sample instant $\mathrm{n}$ can be estimated from the instantaneous angles of the poles using the formula $f_{i, n}=\frac{\arg \left[P_{i, n}\right]}{2 \pi}$ for $\left|P_{i, n}\right| \simeq 1$.

Step6:From time varying parameters $a_{k, n}$ we can predict non stationary signal using (4) with initial conditions $x_{n} ; \mathrm{n}=0,1, \ldots \mathrm{p}$ where $\mathrm{p}$ is the TVAR model order

Step7:The time varying power spectral density P (f; n) can be estimated using(27) .

\section{Simulation Results}

The TVAR model is applied to Linear FM Signal, Non Linear FM Signal. and Closely spaced three component Linear chirp signal. The performance of TVAR modeling approach in the estimation of Instantaneous frequency estimation, signal prediction, time-varying spectra under noisy condition is demonstrated.

\subsection{Linear FM Signal}

In this section, we express TVAR based IF estimation of a signal containing two linear FM components in white noise. The IF laws of the FM components in the signal are

$f_{1, n}=0.05+2(0.0015) n$

$f_{2, n}=0.2+2(0.002) n$ 
We consider a data record of $\mathrm{N}=64$ samples and a sample rate of $1 \mathrm{~Hz}$, hence $\mathrm{n}=0,1,2, \ldots, 63$ and the two FM components chirp in frequency from $0.05 \mathrm{~Hz}$ to $0.239 \mathrm{~Hz}$ and from $0.2 \mathrm{~Hz}$ to $0.452 \mathrm{~Hz}$ correspondingly. The FM Signal generated is specified below

$x_{n}=e^{j 2 \pi[((0.05+(0.0015) n)) n+\varphi]}+e^{j 2 \pi[((0.2+(0.002) n)) n+\varphi]}$

The above signal is corrupted by means of a complex additive white Gaussian noise (AWGN) at $\mathrm{SNR}=20 \mathrm{~dB}$. Compute TVAR model order using MLE algorithm we get $\mathrm{p}=2$ and $\mathrm{q}=3$. Furthermore we use the covariance technique of signal modeling and set $\tau=[p, N-1]$ resulting in $n=2,3, \ldots, 63$. For the covariance technique the time polynomial basis function description is adapted as shown below

$u_{m, n}=\left(\frac{n-p}{N}\right)^{m} \quad m=0,1, \ldots \ldots \ldots \ldots q ; n=p, p+1, \ldots \ldots . . . . .1$.

For $\mathrm{p}=2, \mathrm{q}=3$ and $\mathrm{N}=64$ plot of the above basis function is shown in Figure (1). A plot of the TVAR coefficients $a_{k, n}$ is shown in Figure(2). Trajectory of Time-varying Poles used for a Linear FM Signal is shown in Figure(3). From Figure (3) we observe that the poles are close to the unit circle as anticipated. For every sample instant n, we now come across the angles of the poles and divide by $2 \pi$ to find the IF estimate of the FM components. The true IF \& estimated IF of the FM components are shown in Figure(4). From Figure (4) we observe that the TVAR based technique has resulted in really nice IF estimation. The mean square error (MSE) among the true IF $f_{i, n}$ and estimated IF $\hat{f}_{i, n}$ for $n=2,3, \ldots \ldots \ldots, 63$ is calculated to be $-109.4847 \mathrm{~dB}$ for $\mathrm{i}=1$ and $-103.611 \mathrm{~dB}$ for $\mathrm{i}=2$.we remind here that the estimates are not accessible for the interval $[0, p-1]=[0,1]$ in the covariance technique.

The TVAR coefficients $a_{k, n}$ can also be used to predict the non stationary process $x_{n}$ by means of equation(4).For the covariance technique, the parameters $a_{k, n}$ can be calculated only for $n=2,3, \ldots \ldots .63$. Consequently, the prediction $\hat{x}_{n}$ is also accessible simply for this interval. The samples $x_{0}$ and $x_{1}$ in this simulation illustration are used as initial setting for the time-varying prediction error filter. The original data record $x_{n}$ in addition to the TVAR prediction are shown in Figure (5), and we observe that the TVAR model has effectively predicted $x_{n}$. The average squared prediction error is calculated to be 0.0403 . The timevarying power spectral density is computed using(27).A plot of the time-frequency distribution for the TVAR model is obtained in Figure (6). At every sample instant, the TFD is projected to comprise peaks at the IF estimates at that instant. To demonstrate this, we also illustrate the analogous flat time-frequency view of the TFD in Figure (7). 


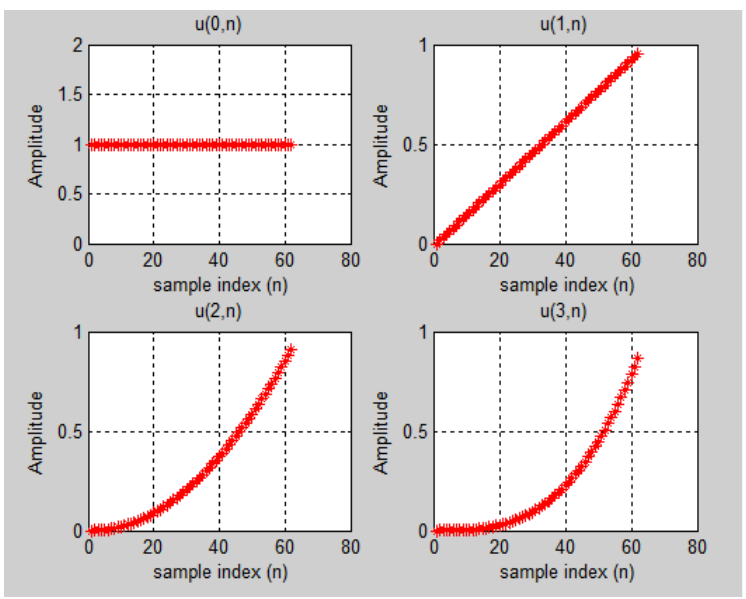

Figure 1.The Time Polynomial Basis Function Set $u_{m, n}$ for $p=2, q=3$

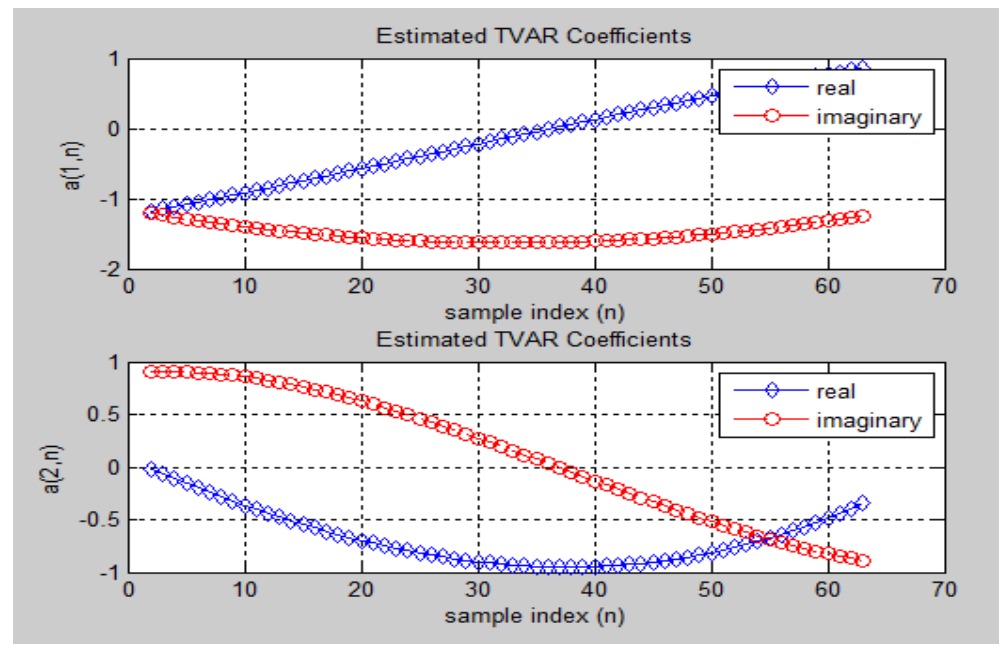

Figure 2. Estimated TVAR Coefficients $a_{k, n}$ used for a Linear FM Signal

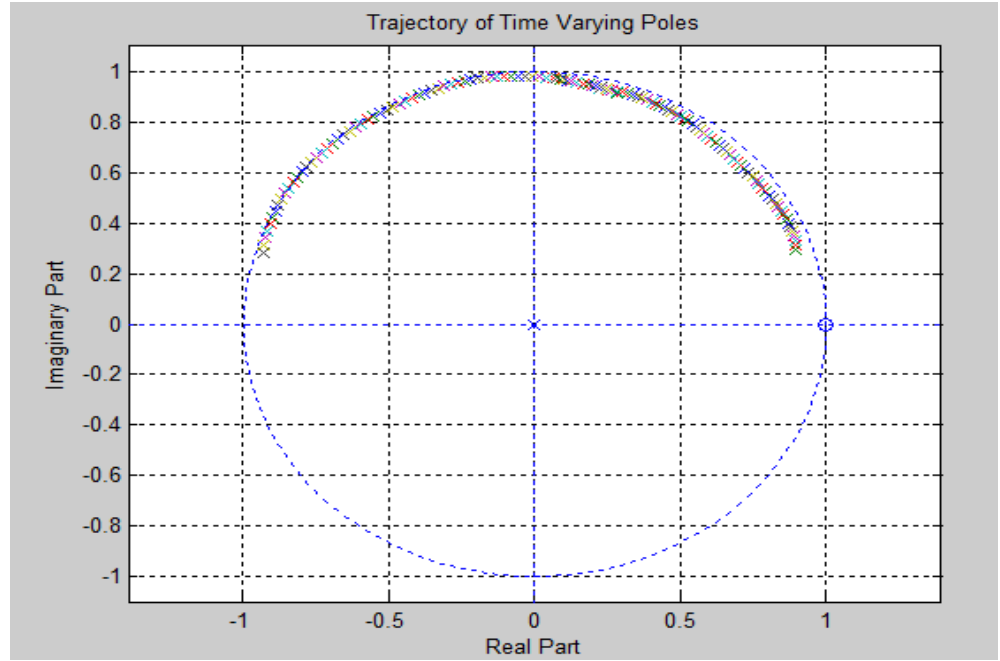

Figure 3. Trajectory of Time-varying Poles used for a Linear FM Signal 


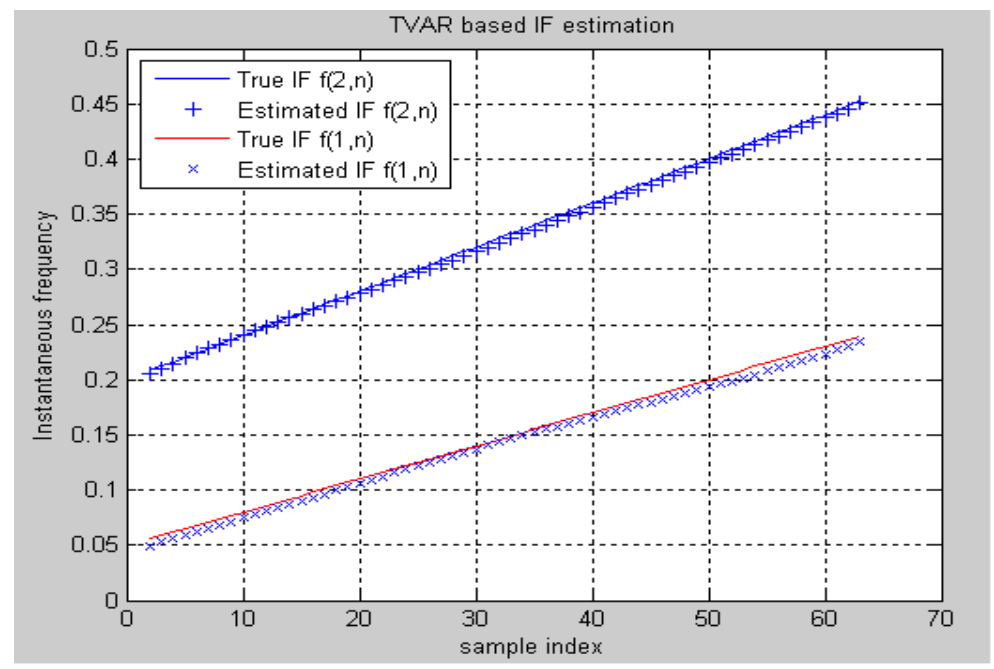

Figure 4. True and Estimated IF of Linear FM Components
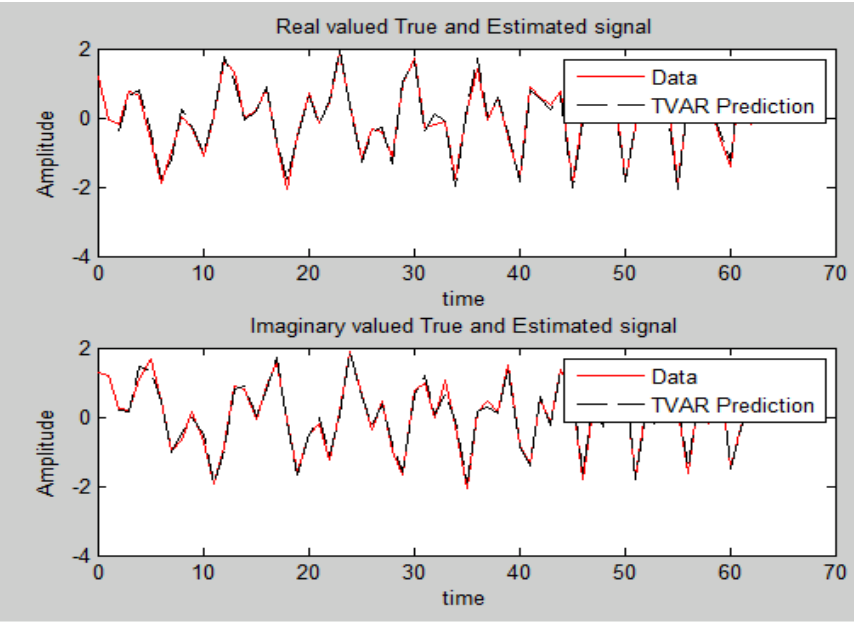

Figure 5. Comparison of Original Data and TVAR Prediction for Linear FM Signal

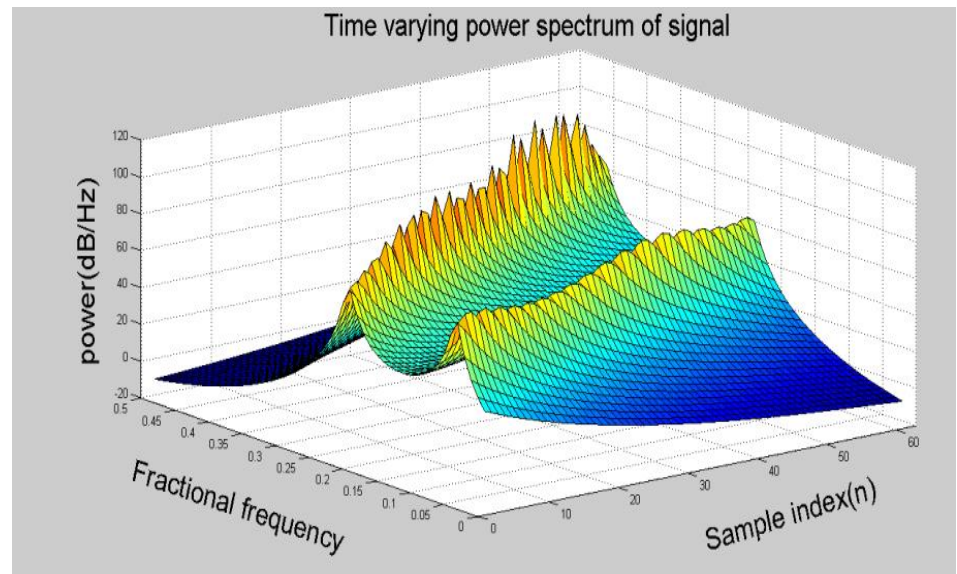

Figure 6. Time Varying Power Spectrum of the TVAR Model of Linear FM Signal 


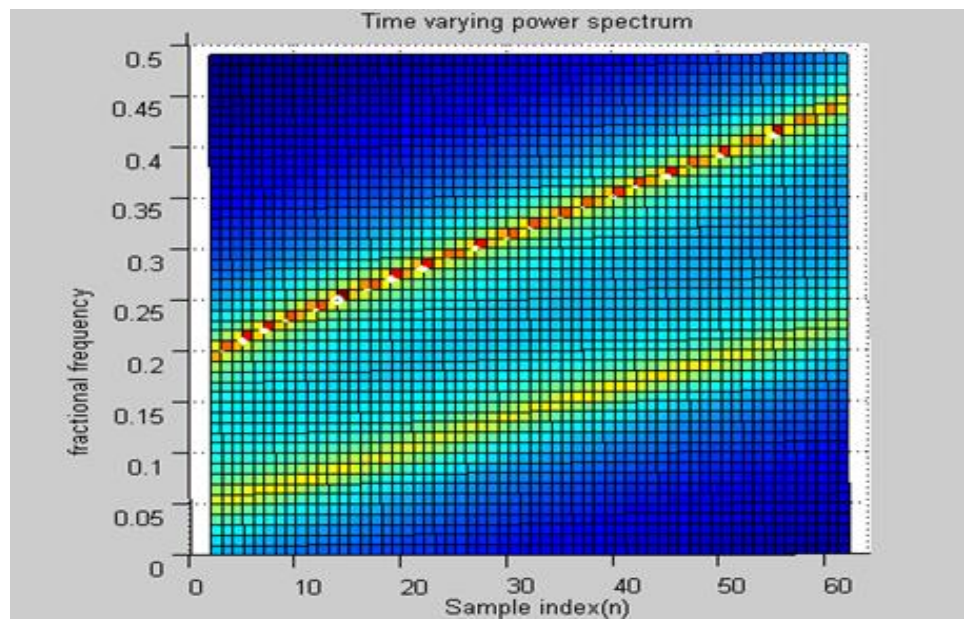

Figure 7. Time-Frequency View of the TFD

\subsection{Non-linear FM Signal}

In this section, we express that the TVAR based IF estimation is extremely useful for realistic situations where the IF law is non-linear. We consider a signal having a single FM component with a sinusoidal varying frequency. The non-linear IF law is specified by,

$$
f_{n}=0.15+0.02(0.1 n \cos (0.1 n)+\sin (0.1 n))
$$

We consider a data record of $\mathrm{N}=64$ samples and a sample rate of $1 \mathrm{~Hz}$.The FM signal generated is specified below,

$x_{n}=\left(e^{j 2 \pi[(0.15+0.02 \sin (0.1 n)) n+\varphi]}\right)$

The above signal is corrupted by means of a complex additive white Gaussian noise (AWGN) at SNR=20dB. Compute the TVAR model order using MLE algorithm we get $\mathrm{p}=2$ and $\mathrm{q}=5$. The basis functions used in this simulation comprise the equivalent form as in the linear FM Signal except that here we have a set of 6 . A plot of the TVAR coefficients $a_{k, n}$ obtained for this signal is shown in Figure(8).A plot of the trajectory of time-varying poles is shown in Figure(9).From Figure(9) we observe that poles corresponding to noise are randomly distributed, typically away from the unit circle while poles that corresponding to the FM components are close to unit circle. The true and estimated IF of the FM component is shown in Figure (10). From Figure (10), we observe that the TVAR based technique have resulted in really nice IF estimation. The mean square error (MSE) among the true IF $f_{n}$ and estimated IF $\hat{f}_{n}$ for $n=2,3, \ldots \ldots \ldots . .63$ is calculated to be $-64.365 \mathrm{~dB}$.

The original data record $x_{n}$ and the TVAR Prediction are shown in Figure (11). The Figure shows successful prediction of $x_{n}$ with an Average square prediction error $=0.0046$. The timevarying power spectral density is computed using(27).A plot of the time-frequency distribution for the TVAR model is obtained in Figure (12). A time-frequency outlook of the TFD is shown in Figure(13)to demonstrate that the TFD peaks are reliable with the estimated IF and the true IF given way in Figure(10). 


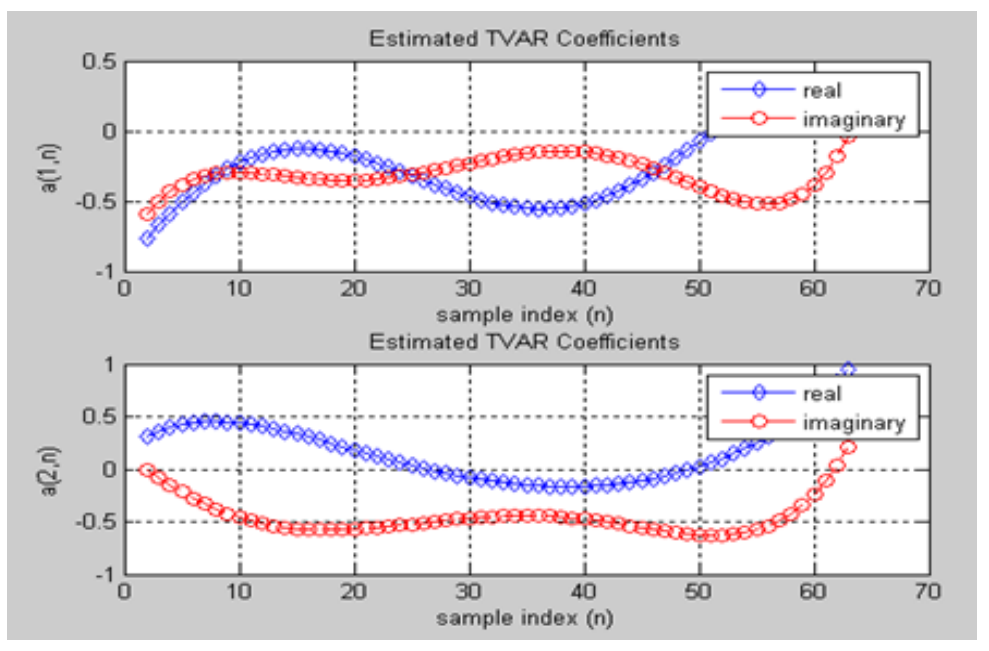

Figure 8. Estimated TVAR Coefficients $a_{k, n}$ for Non-linear FM Signal

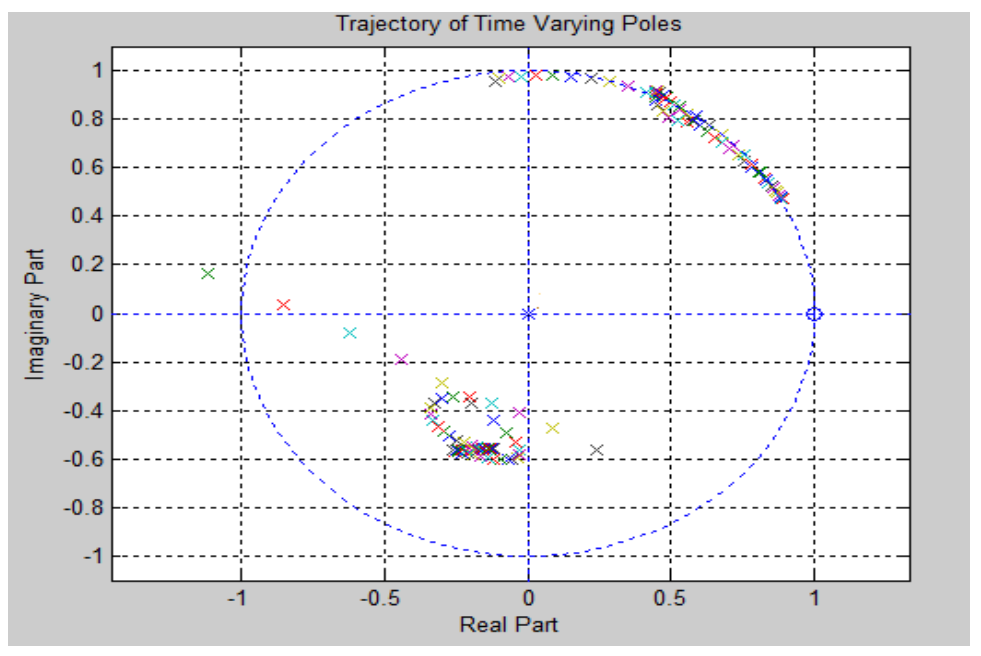

Figure 9. Trajectory of Time-varying Poles used for a Non-linear FM Signal

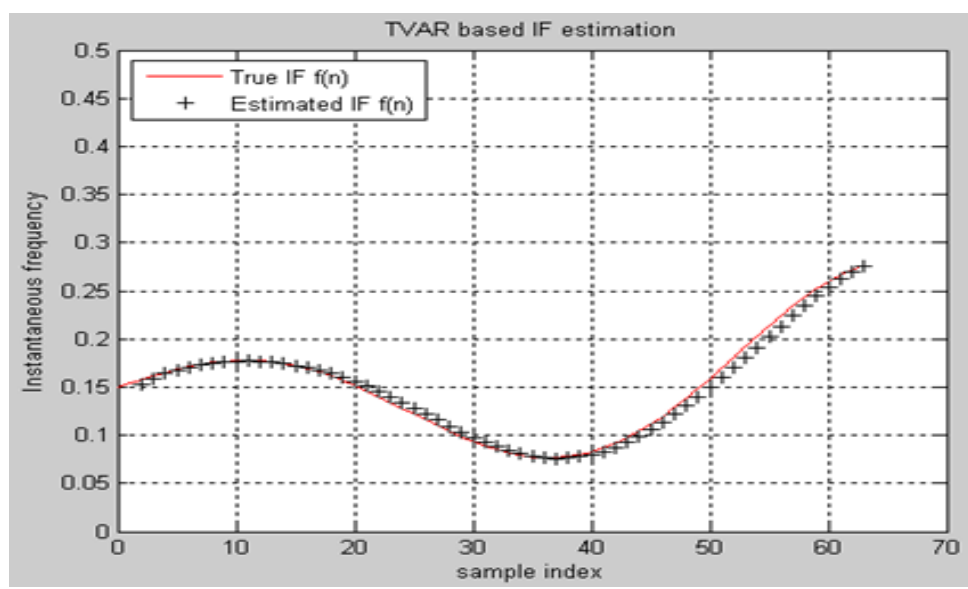

Figure 10. True and Estimated IF of Non-linear FM Component 


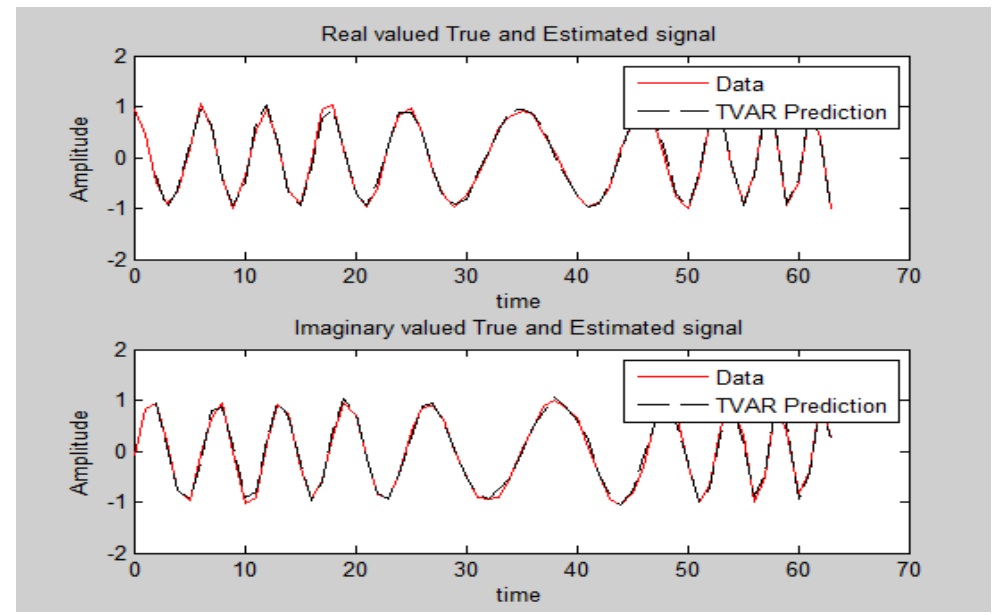

Figure11. Comparison of TVAR Prediction and Original Data for Non-linear FM Signal

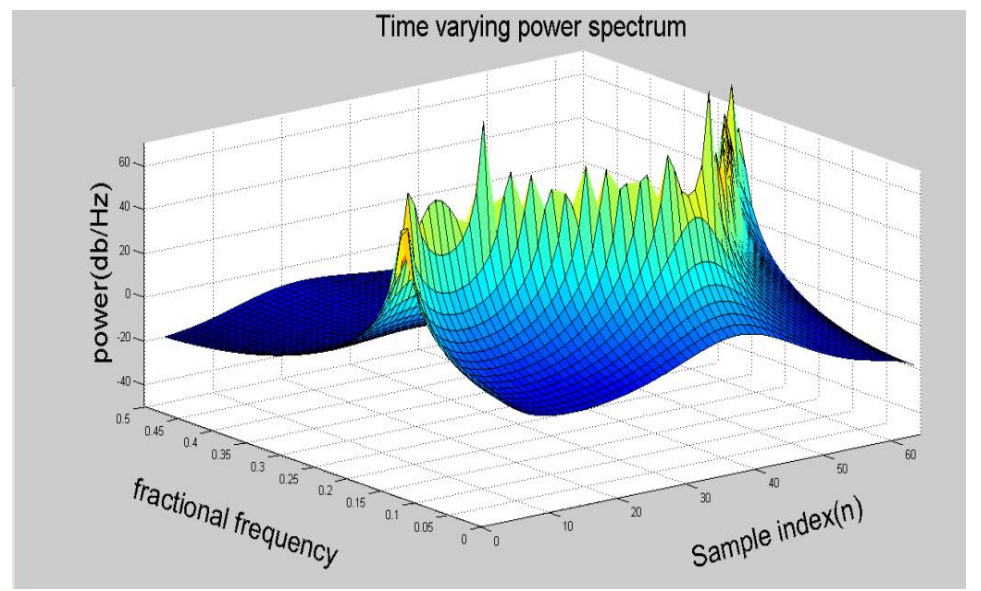

Figure 12. Time Varying Power Spectrum of the TVAR Model of Non- linear FM Signal

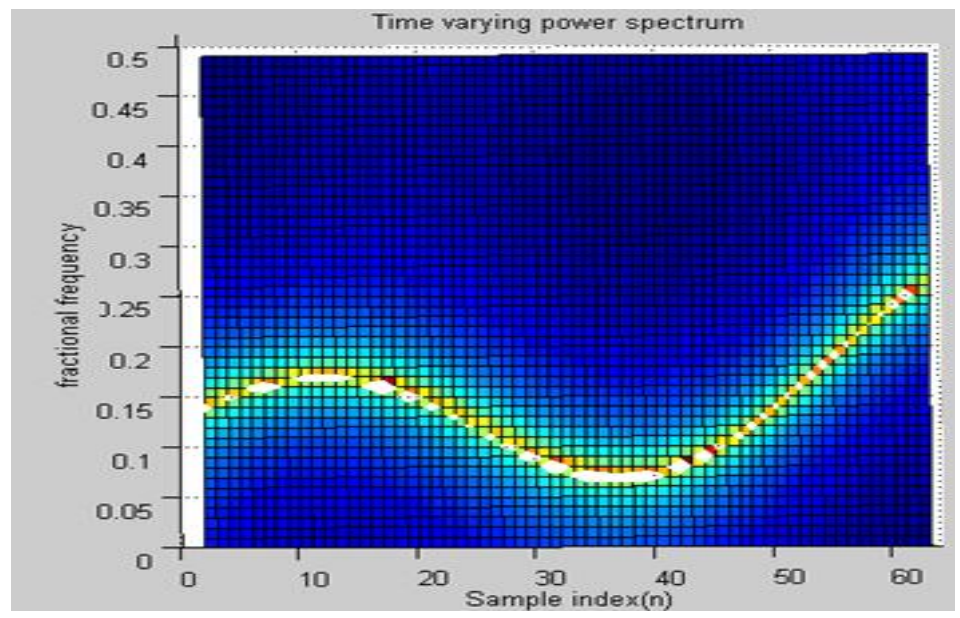

Figure 13.Time-Frequency View of the TFD of Non-linear FM Signal 


\subsection{Closely Spaced Three Component Linear Chirp Signal}

In this section we have demonstrated the ability of TVAR Model to resolve closely spaced spectral peaks under noisy conditions. The simulated signal is composed of three linear chirp components of which the two components are spaced very close in time-frequency plane. The last component is of amplitude modulated (AM) signal.

$$
\begin{aligned}
& x_{n}=\sin \left(2 \pi\left(\mathrm{f}_{1}+a \mathrm{n}\right) \mathrm{n}\right)+\sin \left(2 \pi\left(\mathrm{f}_{2}+a \mathrm{n}\right) \mathrm{n}\right)+\sin (2 \pi \mathrm{n}) \sin \left(2 \pi\left(\mathrm{f}_{3}+a \mathrm{n}\right) \mathrm{n}\right) \\
& \mathrm{n}=0,1,2, \ldots \ldots, 255 . \\
& a=100, \mathrm{f}_{1}=100 \mathrm{~Hz}, \mathrm{f}_{2}=200 \mathrm{~Hz}, \mathrm{f}_{3}=250 \mathrm{~Hz}, \mathrm{~F}_{\mathrm{s}}=1000 \mathrm{~Hz} .
\end{aligned}
$$

Instantaneous frequency Law of first component $\quad \mathrm{F}_{\mathrm{s} 1}=\mathrm{f}_{1}+2 * a * \mathrm{n}$,

Instantaneous frequency Law of second component $\mathrm{F}_{\mathrm{s} 2}=\mathrm{f}_{2}+2 * a * \mathrm{n}$,

Instantaneous frequency Law of third component $\mathrm{F}_{\mathrm{s} 3}=\mathrm{f}_{3}+2 * a * \mathrm{n}$,

The above signal is corrupted by means of a complex additive white Gaussian noise (AWGN) at SNR=20 dB. Compute TVAR model order using MLE algorithm we get $\mathrm{p}=6$ and $\mathrm{q}=7$. Furthermore we use the covariance technique of signal modeling and set $\tau=[p, N-1]$ resulting in $n=6,7, \ldots, 255$. The basis functions used in this simulation comprise the equivalent form as in the linear FM Signal except that here we have a set of 8 .

TVAR coefficients $a_{1, n} a_{2, n}$ of this signal is shown in Figure (14). Trajectory of time varying poles are shown in Figure (15).From Figure (15)we observe that poles are complex conjugate to each other(since the signal is real )and also half of the poles are lie in the upper half of Z-plane and remaining half of the poles are lower half of the Z-plane. For IF estimation consider the poles that lie either upper half (or) lower half of the Z-plane. The true IF \& estimated IF of the composite signal is shown in Figure (16). From Figure (16) we observe that the TVAR based technique has resulted in really nice IF estimation. The mean square error (MSE) among the true IF $f_{i, n}$ and estimated IF $\hat{f}_{i, n}$ for $n=6,7 \ldots \ldots \ldots, 255$ is calculated to be $-108.5078 \mathrm{~dB}$ for $\mathrm{i}=1$ and $-92.427 \mathrm{~dB}$ for $\mathrm{i}=2$, and $-76.743 \mathrm{~dB}$ for $\mathrm{i}=3$, we remind here that the estimates are not accessible for the interval $[0, p-1]=[0,5]$ in the covariance technique.

From TVAR coefficients we can predict the signal using (4) and the result is shown in Figure (17),we observe that predicted signal is very close to true signal. The average squared prediction error is calculated to be 0.1773 . From TVAR parameters we can also estimate the Time-varying power spectrum using (27) is shown Figure (18).At every sample instant, the time-frequency distribution (TFD) is projected to comprise peaks at the IF estimates of that instant. To demonstrate this, we also illustrate the corresponding flat time-frequency view of the TFD in Figure (19). Figure (19) shows TVAR model approach have been used effectively for resolving closely spaced spectral peaks (super-resolution spectral estimation)in the composite signal 

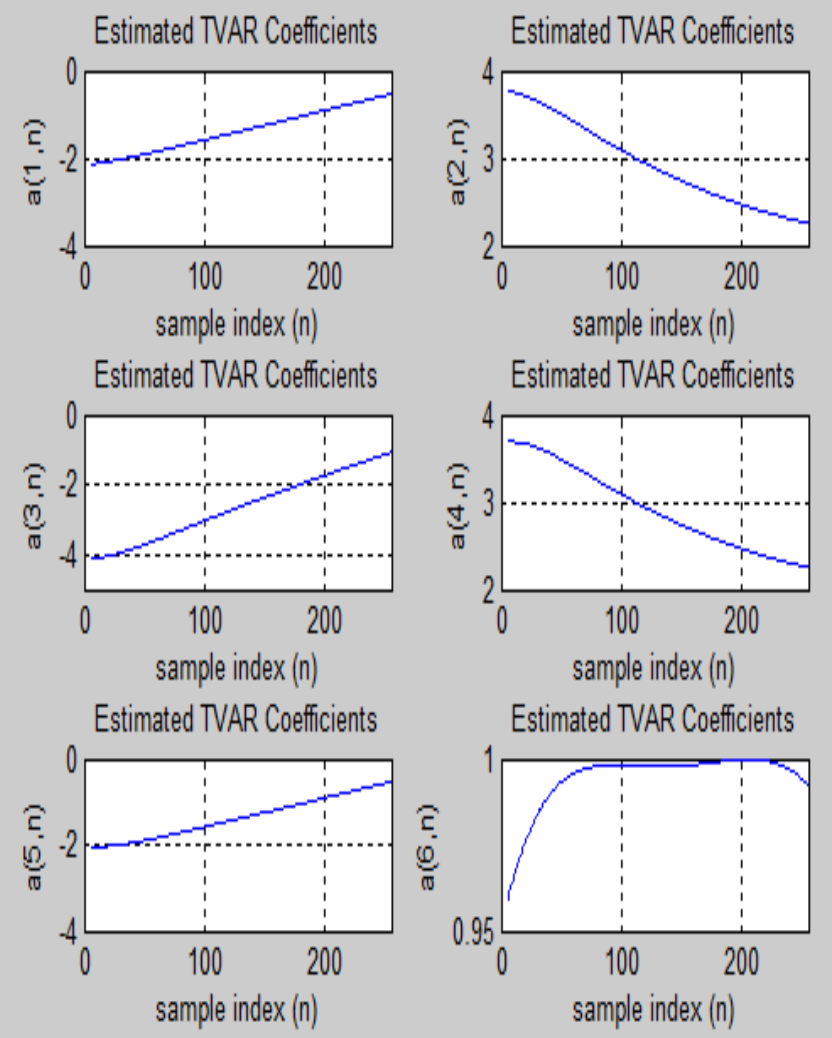

Figure 14. Estimated TVAR Coefficients $a_{k, n}$ for Three Component Chirp Signal

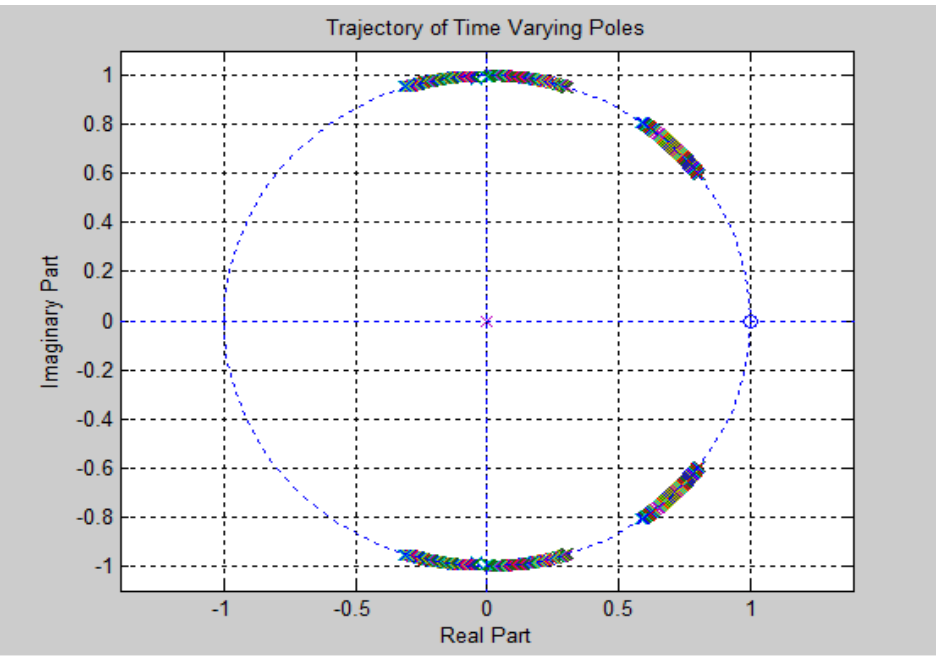

Figure 15. Trajectory of Time-varying Poles used for Three Component Linear Chirp Signal 


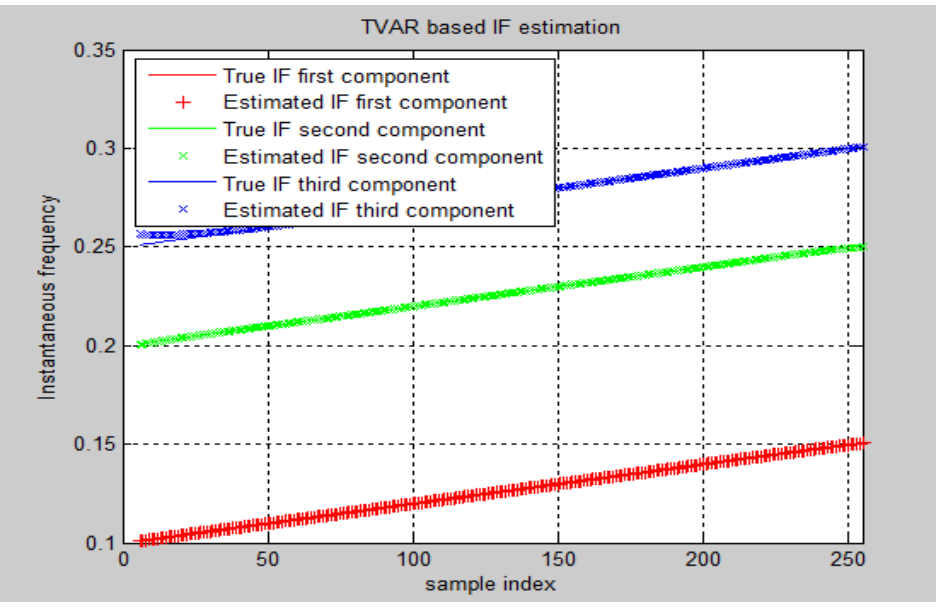

Figure 16. True and Estimated IF of Three Component Linear Chirp Signal

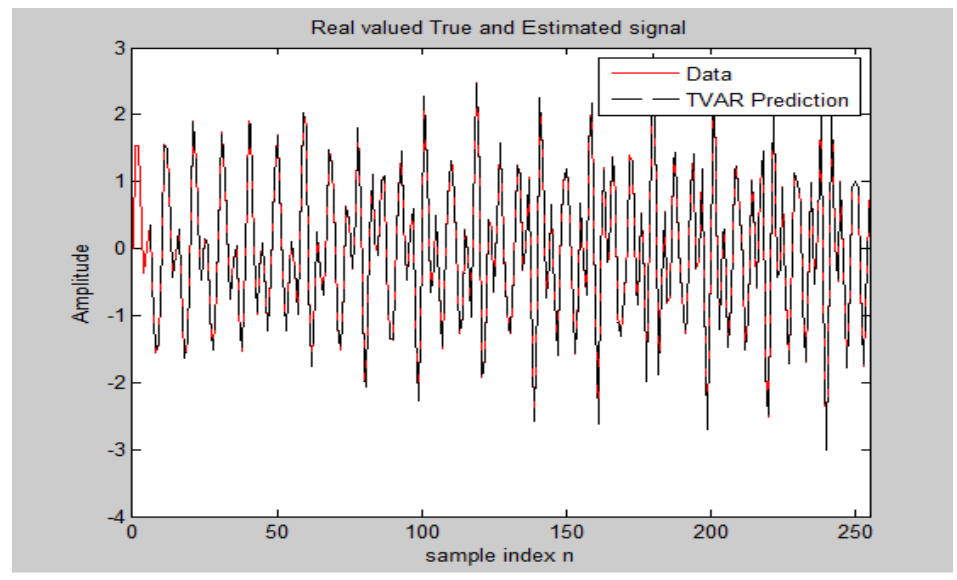

Figure17. Comparison of TVAR Prediction and Original Data for Three Component Linear Chirp Signal

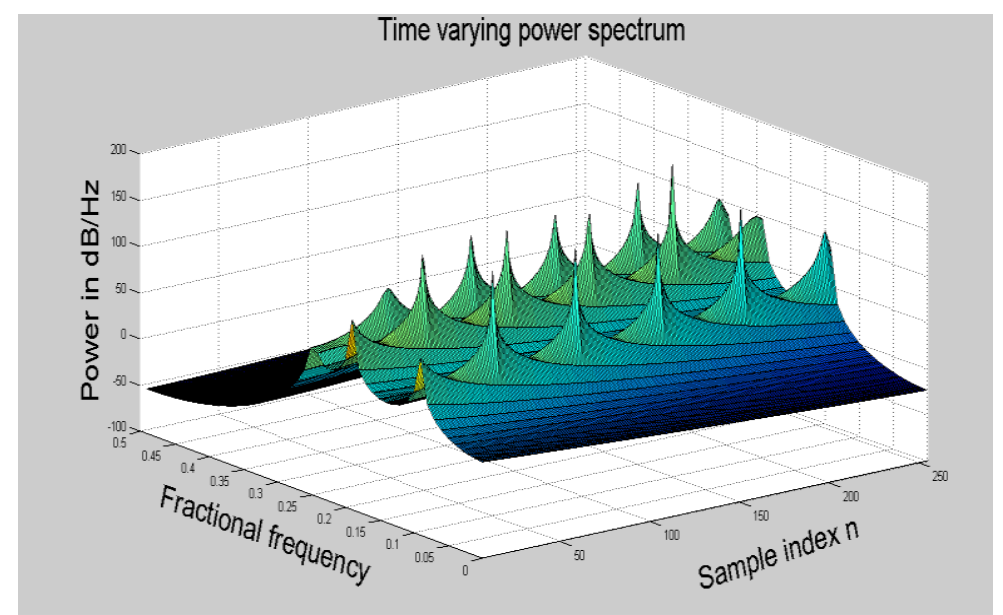

Figure 18. Time Varying Power Spectrum of the TVAR Model of Three Component Linear Chirp Signal 


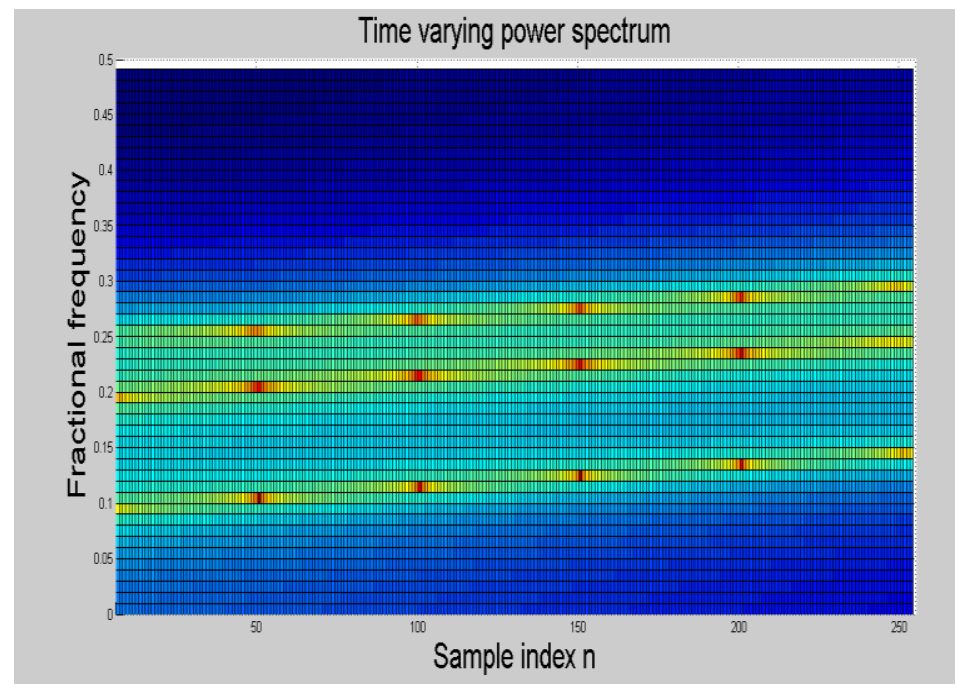

\section{Figure 19. Time-Frequency View of the TFD of Three Component Linear Chirp} Signal

\section{Conclusions}

In this paper we presented the IF estimation method based on TVAR model and few example simulations to demonstrate that this technique works well for linear and non-linear IF laws in addition to small data records. We have presented Non stationary signal prediction and power spectrum estimation of Non stationary signals. We have also demonstrated the ability of TVAR Model to resolve closely spaced spectral peaks. Simulations demonstrate that TVAR model provides better resolution, accuracy and noise resistance.

\section{References}

[1] B. Boashash, "Estimating and interpreting the instantaneous frequency of a signal.ii.Algorithms and applications", Proceedings of the IEEE, vol. 80, no. 4, (1992) April, pp. 540-568.

[2] P. Shan and A. A. (Louis) Beex, "High-resolution instantaneous frequency estimation based on time-varying AR modeling", IEEE-SP International Symposium on Time-Frequency and Time-Scale Analysis, (1998) October, pp. 109-112.

[3] Y. Grenier, "Time-dependent ARMA modeling of nonstationary signals", IEEETransactions on coustics, Speech, and Signal Processing, vol. 31, no. 4, (1983) August, pp. 899-911.

[4] T. S. Rao, "The fitting of non-stationary time-series models with time-dependent parameters", Journal of the Royal Statistical Society. Series B (Methodological), vol. 32,no. 2, (1970), pp. 312-322.

[5] L. A. Liporace, "Linear estimation of nonstationary signals", Journal of the Acoustical Society of America, vol. 58, no. 6, (1975) December, pp. 1288-1295.

[6] M. Hall, A. V. Oppenheim and A. Willsky, "Time-varying parametric modeling of speech", IEEE Conference on Decision and Control, vol. 16, no. 1, (1977) December, pp. 1085-1091.

[7] K. C. Sharman and B. Friedlander, "Time-varying autoregressive modeling of a class of nonstationary signals", IEEE Conference on Acoustics, Speech, and Signal Processing, vol. 9, no. 1, (1984) March, pp. 227-230.

[8] K. B. Eom, "Analysis of Acoustic Signatures from Moving Vehicles Using Time-Varying Autoregressive Models", Multisignal Systems and Signal Processing, vol. 10, (1999), pp. 357-378.

[9] A. A. (Louis) Beex and P. Shan, "A time-varying Prony method for instantaneous frequency estimation at low SNR”, IEEE International Symposium on Circuits and Systems, vol. 3, (1999) May, pp. 3-8.

[10] R. Kadanna Pally and A. A. (Louis) Beex, "Modeling of time-varying instantaneous frequency in a finitely correlated environment", 16th International Conference on Digital Signal Processing, Santorini, Greece, ppr T3C.3, (2009) July.

[11] A. A. (Louis) Beex and P. Shan, "A time-varying Prony method for instantaneous frequency estimation at low SNR”, IEEE International Symposium on Circuits and Systems, vol. 3, (1999) May, pp. 3-8. 
[12] S. Mukhopadhyay and P. Sircar, "Parametric modeling of nonstationary signals: A unified approach", Signal Processing, vol. 60, (1997), pp. 135-152.

[13] R. Kadanna Pally, "Implemeentation of instantaneous frequency estimation based on time-varying AR Modeling", M.S.Thesis, Virginia Tech, (2009) April.

[14] M. Neidzwiecki, "Identification of Time-varying Processes", John Wiley \& Sons, Chicester, England, (2000).

[15] L. Cohen, “Time-frequency Distributions-A Review”, vol. 77, no. 7, (1989), pp. 941-981.

[16] H. I. Choi and W. J. Williams, "Improved Time-Frequency Representation of Multicomponent Signals Using Exponential Kernel”, IEEE Trans.on Acoustics,Speech and Signal Processing, vol. 17, no. 6, (1989).

[17] W. Martin and P. Flandrin, "Wigner-Ville Spectral Analysis of Non-stationary Processes", IEEE Trans on Acoustics,Speech and Signal Processing, vol. 33, (1985), pp. 1461-1470.

[18] S. M. Kay, "Modern Spectral Estimation:Theory and Application", Prentice-Hall, Englewood Cliffs, NJ, (1988).

\begin{abstract}
Authors
G. Ravi Shankar Reddy is prsently working as an Associate Professor, CVR Engineering College of Engineering ,Hyderabad,India .He received the B.Tech degree in Electronics and Communications Engineering from Jawaharlal Nehru Technological University,Hyderabad,India,in 2001.He received the M.Tech degree in Systems and Signal Processing from Osmania University,Hyderabad,India in 2004. Presently he is pursuing his Ph.D. from Jawaharlal Nehru Technological University,Ananatapur,India.Under the guidence of Dr.Rameshwar Rao.His research area is Nonstationary Signalprocessing.
\end{abstract}

Dr.Rameshwar Rao is currently Vice Chancellor of Jawaharlal Nehru Technological University, Hyderabad, India. He guided several PhD students in the area of VLSI and Signal processing. He has more than 50 publications in various National and International Journals. His research interests are in VLSI and Signalprocessing. 
International Journal of Signal Processing, Image Processing and Pattern Recognition Vol.7, No.2 (2014) 